\title{
Commentary on "Sustained versus Standard Inflations during Neonatal Resuscitation to Prevent Mortality and Improve Respiratory Outcomes"
}

\author{
Nicolas A. Bamat ${ }^{\mathrm{a}}$ Roger F. Soll ${ }^{\mathrm{b}}$ \\ ${ }^{a}$ Children's Hospital of Philadelphia and Perelman School of Medicine, University of Pennsylvania, \\ Philadelphia, PA, USA; ' Larner College of Medicine, University of Vermont, Burlington, VT, USA
}

\section{Commentary}

After birth, the newborn lungs must rapidly transition from a fluid- to a gas-filled organ to achieve effective gas exchange. Clearance of fetal lung fluid occurs less readily in preterm newborns, contributing to heterogeneous lung aeration and injury. This may help propagate a cascade of harm that contributes to chronic lung disease, neurodevelopmental impairment, and death. The potential value of a therapy to mitigate this early injurious cascade is considerable and explains the enthusiasm around the promise of sustained inflations (SI).

Intermittent positive pressure ventilation (IPPV) is the standard of care in preterm newborns requiring ventilatory support at birth. Compared to IPPV, SI deliver a longer peak distending pressure. When applied in the initial resuscitative steps, SI theoretically hasten fetal lung clearance and facilitate more effective and less injurious homogenous ventilation. Elegant animal studies strongly support the benefit of SI for establishing lung volumes and facilitating gas exchange without adverse circulatory effects $[1,2]$. Randomized controlled trials sought to confirm that these observations translated to clinical benefit in humans.

\footnotetext{
karger@karger.com

(c) 2021 S. Karger AG, Basel

www.karger.com/neo

Karger
}

In the recently published Cochrane review which is the focus of this commentary (abstract below), Bruschettini and colleagues [3] update their synthesis of data from trials randomizing newborns to either IPPV or SI. Importantly, this now includes data from the recently completed SAIL trial [4], the largest to date. The authors identified 10 eligible trials and pooled data from 9 trials including 1,467 subjects; a small study that evaluated SI with chest compressions was considered separately [5]. In meta-analysis, the superiority of SI over IPPV could not be confirmed for the outcomes of pneumothorax, use of mechanical ventilation, chronic lung disease, or death before discharge. In fact, the point estimate for death favored IPPV (Table 1). The authors concluded that evidence to support the effectiveness of SI in human newborns is lacking.

Is it fair to question the generalizability of these findings? Are there specific applications of SI that do, in fact, lead to benefit? After all, the pooled trials differ in the number, magnitude, and duration of SI. Further, while some protocols only applied SI as "rescue therapy" in newborns requiring resuscitation, others gave it "prophylactically" in all newborns. Have we identified and adequately examined the best approach? There is also sub- 
Table 1. Summary of findings: pooled results comparing SI to IPPV in newborns undergoing resuscitation after birth

\begin{tabular}{|c|c|c|c|c|c|}
\hline \multirow[t]{2}{*}{ Outcome } & \multirow{2}{*}{$\begin{array}{l}\text { Participants, } \\
n \text { (studies) }\end{array}$} & \multicolumn{2}{|c|}{ Events/total, $n / N(\%)$} & \multirow{2}{*}{$\begin{array}{l}\text { Relative risk } \\
(95 \% \mathrm{CI})\end{array}$} & \multirow{2}{*}{$\begin{array}{l}\text { Quality of } \\
\text { evidence } \\
\text { (GRADE) }\end{array}$} \\
\hline & & SI & IPPV & & \\
\hline Pneumothorax & $1,458(9)$ & $34 / 720(4.7)$ & $35 / 657(5.3)$ & $0.89(0.57-1.39)$ & Low \\
\hline Mechanical ventilation & $910(4)$ & $189 / 463(40.8)$ & $206 / 447(46.1)$ & $0.89(0.77-1.02)$ & Moderate \\
\hline Chronic lung disease & $1,418(8)$ & $218 / 741(29.4)$ & $203 / 677(30.0)$ & $0.98(0.84-1.13)$ & Moderate \\
\hline Death before discharge & $1,458(9)$ & $95 / 763(12.4)$ & $78 / 695(11.2)$ & $1.09(0.83-1.43)$ & Moderate \\
\hline
\end{tabular}

SI, sustained inflations; IPPV, intermittent positive pressure ventilation.

stantial heterogeneity in the gestational age of enrolled subjects, with only 3 of the 9 trials predominantly enrolling extremely low-birth-weight infants $[4,6,7]$. Have we identified and adequately studied the right population? In a separate recent systematic review, Foglia and colleagues [8] examine whether the impact of SI differ when prophylactic is compared to rescue therapy, or varying gestational age categories are considered separately. In neither instance was the effectiveness of SI confirmed for any specific subgroup.

Why have the promising findings from animal studies failed to translate into human benefit? Various factors may contribute. There may be discordance in the transitional physiology of preterm humans compared to sheep or rabbits, resulting in differential SI effects. Alternatively, there may be discordance in the experimental environment. The average effect of SI reliably delivered through an endotracheal tube in anesthetized animals is likely to differ from the effects of SI delivered via face mask to newborns by a diverse group of teams operating in lesscontrolled clinical environments. It is also worth noting that the animal and human studies did not measure similar outcomes. The former focused on immediate physiologic and imaging measures infrequently available in human trials. Had similar measures been available in human trials, would they have revealed that newborns do not experience similar improvements in markers of transitional physiology, or a lack of clinical benefit despite improvements in these surrogate outcomes?
The idea that a safely applied open-lung ventilation strategy can improve outcomes in preterm newborns should not be abandoned. The possibility that SI can be a useful tool in implementing this strategy under the right circumstances remains. However, the findings to date will appropriately encourage considerable scrutiny. As highlighted by Bruschettini and colleagues [3] in their concluding remarks, any future studies should choose participants carefully, strongly consider more detailed monitoring of immediate physiologic effects, and measure long-term neurodevelopmental outcomes.

\section{Acknowledgement}

Cochrane Neonatal Reviews are produced with support from the Vermont Oxford Network, a worldwide collaboration of health professionals dedicated to providing evidence-based care of the highest quality for newborn infants and their families.

\section{Conflict of Interest Statement}

The authors serve in the editorial group of Cochrane Neonatal.

\section{Funding Sources}

None relevant. 


\section{References}

1 Sobotka KS, Hooper SB, Allison BJ, te Pas AB, Davis PG, Morley CJ, et al. An initial sustained inflation improves the respiratory and cardiovascular transition at birth in preterm lambs. Pediatr Res. 2011;70(1):56-60.

2 te Pas AB, Siew M, Wallace MJ, Kitchen MJ, Fouras A, Lewis RA, et al. Establishing functional residual capacity at birth: the effect of sustained inflation and positive end-expiratory pressure in a preterm rabbit model. Pediatr Res. 2009;65(5):537-41.

3 Bruschettini M, O’Donnell CPF, Davis PG, Morley CJ, Moja L, Calevo MG. Sustained versus standard inflations during neonatal resuscitation to prevent mortality and improve respiratory outcomes. Cochrane Database Syst Rev. 2020(3):CD004953.
4 Kirpalani H, Ratcliffe SJ, Keszler M, Davis PG, Foglia EE, te Pas A, et al. Effect of sustained inflations vs intermittent positive pressure ventilation on bronchopulmonary dysplasia or death among extremely preterm infants: the SAIL randomized clinical trial. JAMA. 2019;321(12):1165-75.

5 Schmölzer GM, O Reilly M, Fray C, van Os S, Cheung P-Y. Chest compression during sustained inflation versus 3:1 chest compression: ventilation ratio during neonatal cardiopulmonary resuscitation: a randomised feasibility trial. Arch Dis Child Fetal Neonatal Ed. 2018;103(5):F455-60.
6 Lindner W, Högel J, Pohlandt F. Sustained pressure-controlled inflation or intermittent mandatory ventilation in preterm infants in the delivery room? A randomized, controlled trial on initial respiratory support via nasopharyngeal tube. Acta Paediatr. 2005;94(3): 303-9.

7 Lista G, Boni L, Scopesi F, Mosca F, Trevisanuto $\mathrm{D}, \mathrm{Messner} \mathrm{H}$, et al. Sustained lung inflation at birth for preterm infants: a randomized clinical trial. Pediatrics. 2015;135(2): e457-64

8 Foglia EE, te Pas AB, Kirpalani H, Davis PG, Owen LS, van Kaam AH, et al. Sustained inflation vs standard resuscitation for preterm infants: a systematic review and meta-analysis. JAMA Pediatr. 2020;174(4):e195897.

(c) 2021 S. Karger AG, Basel

\section{Cochrane Abstract}

Background: At birth, infants' lungs are fluid filled. For newborns to have a successful transition, this fluid must be replaced by air to enable gas exchange. Some infants are judged to have inadequate breathing at birth and are resuscitated with positive pressure ventilation (PPV). Giving prolonged (sustained) inflations at the start of PPV may help clear lung fluid and establish gas volume within the lungs. Objectives: To assess the benefits and harms of an initial sustained lung inflation (SLI) (>1 s duration) versus standard inflations ( $\leq 1 \mathrm{~s}$ ) in newborn infants receiving resuscitation with intermittent PPV. Search Methods: We used the standard search strategy of Cochrane Neonatal to search the Cochrane Central Register of Controlled Trials (CENTRAL; 2019, Issue 3), MEDLINE via PubMed (1966 to April 1, 2019), Embase (1980 to April 1, 2019), and the Cumulative Index to Nursing and Allied Health Literature (CINAHL) (1982 to April $1,2019)$. We also searched clinical trials databases, conference proceedings, and the reference lists of retrieved articles to identify randomized controlled trials (RCTs) and quasirandomized trials. Selection Criteria: RCTs and quasi-RCTs comparing initial SLI versus standard inflations given to infants receiving resuscitation with PPV at birth. Data Collection and Analysis: We assessed the methodological quality of included trials using Cochrane Effective Practice and Organization of Care Group (EPOC) criteria (assessing randomization, blinding, loss to follow-up, and handling of outcome data). We evaluated treatment effects using a fixed-effect model with risk ratio (RR) for categorical data and mean standard deviation (SD) and weighted mean difference (WMD) for continuous data. We used the GRADE approach to assess

Sustained versus Standard Inflations during Neonatal Resuscitation the quality of evidence. Main Results: Ten trials enrolling 1,467 infants met our inclusion criteria. Investigators in 9 trials (1,458 infants) administered sustained inflation with no chest compressions. Use of sustained inflation had no impact on the primary outcomes of this review: mortality in the delivery room (typical RR 2.66, 95\% confidence interval [Cl]: $0.11-63.40\left[I^{2}\right.$ not applicable]; typical RD $0.00,95 \% \mathrm{Cl}:-0.02$ to $0.02 ; P^{2}=0 \% ; 5$ studies, 479 participants) and mortality during hospitalization (typical RR 1.09, 95\% Cl: 0.83-1.43; $R^{2}=42 \%$; typical RD $0.01,95 \% \mathrm{Cl}:-0.02$ to $0.04 ; R^{2}=24 \%$; 9 studies, 1,458 participants). The quality of the evidence was low for death in the delivery room because of limitations in study design and imprecision of estimates (only one death was recorded across studies). For death before discharge, the quality was moderate: with longer follow-up, there were more deaths $(n=143)$, but limitations in study design remained. Among secondary outcomes, duration of mechanical ventilation was shorter in the SLI group (mean difference $[\mathrm{MD}]-5.37$ days, $95 \% \mathrm{Cl}:-6.31$ to $-4.43 ; I^{2}=95 \% ; 5$ studies, 524 participants; low-quality evidence). Heterogeneity, statistical significance, and magnitude of effects of this outcome are largely influenced by a single study at high risk of bias: when this study was removed from the analysis, the size of the effect was reduced (MD -1.71 days, $95 \% \mathrm{Cl}:-3.04$ to $\left.-0.39 ; P^{2}=0 \%\right)$. Results revealed no differences in any of the other secondary outcomes (e.g., risk of endotracheal intubation outside the delivery room by $72 \mathrm{~h}$ of age [typical RR $0.91,95 \% \mathrm{Cl}: 0.79-1.04 ; R^{2}=65 \% ; 5$ studies, 811 participants]; risk of surfactant administration during hospital admission [typical RR 0.99, 95\% Cl: 0.91-1.08; $P^{2}=0 \%$; 9 studies, 1,458 
participants]; risk of chronic lung disease [typical RR 0.99, 95\% Cl: 0.83-1.18; $I^{2}=0 \% ; 4$ studies, 735 participants]; pneumothorax [typical RR $0.89,95 \% \mathrm{Cl}: 0.57-1.40 ; R^{2}=34 \%$; 8 studies, 1,377 infants]; or risk of patent ductus arteriosus requiring pharmacological treatment [typical RR 0.99, 95\% Cl: $0.87-1.12 ; P^{2}=48 \% ; 7$ studies, 1,127 infants]). The quality of evidence for these secondary outcomes was moderate (limitations in study design - GRADE) except for pneumothorax (low quality: limitations in study design and imprecision of estimates - GRADE). We could not perform any metaanalysis in the comparison of the use of initial sustained inflation versus standard inflations in newborns receiving resuscitation with chest compressions because we identified only one trial for inclusion (a pilot study of 9 preterm infants). Authors' Conclusions: Our meta-analysis of 9 studies shows that sustained lung inflation without chest compression was not better than intermittent ventilation for reducing mortality in the delivery room (low-quality evidence-GRADE) or during hospitalization (moderate - quality evidence - GRADE), which were the primary outcomes of this review. However, the single largest study, which was well conducted and had the greatest number of enrolled infants, was stopped early for higher mortality rate in the sustained inflation group. When considering secondary outcomes, such as rate of intubation, rate or duration of respiratory support, or bronchopulmonary dysplasia, we found no benefit of sustained inflation over intermittent ventilation (moderate quality evidence - GRADE). Duration of mechanical ventilation was shortened in the SLI group (low-quality evidence - GRADE); this result should be interpreted cautiously, however, as it might have been influenced by study characteristics other than the intervention. There is no evidence to support the use of sustained inflation based on evidence from our review. 